\title{
ERRATUM
}

\section{Exact solutions of the spherically symmetric gravitational field equations}

\section{HU He-sheng}

Institute of Mathematics, Fudan University, Shanghai 200433, China

(C) Higher Education Press and Springer-Verlag 2006

Front. Math. China (2006) 2: $169-177$

Due to typesetting production errors, some mistakes were made. The Editorial Office regrets these errors.

(i) Some of the equation numbers have been in confusion. Here is the erratum:

\begin{tabular}{cc||cc}
\hline error & correct & error & correct \\
\hline$(1.1)$ & $(1)$ & $(2.13)$ & $(14)$ \\
$(2.3)$ & $(4)$ & $(3.18)$ & $(21)$ \\
$(2.4)$ & $(5)$ & $(3.19)$ & $(22)$ \\
$\ldots$ & $\cdots$ & $(3.20)$ & $(23)$ \\
$(2.12)$ & $(13)$ & $(3.21)$ & $(25)$ \\
\hline
\end{tabular}

(ii) In the theorem on page 174: (13)-(15) should be (13)-(14); (16)-(18) should be (16)-(17); (18)-(19) should be (19)-(20).

(iii) Figure 2 on page 177 should be the following one:

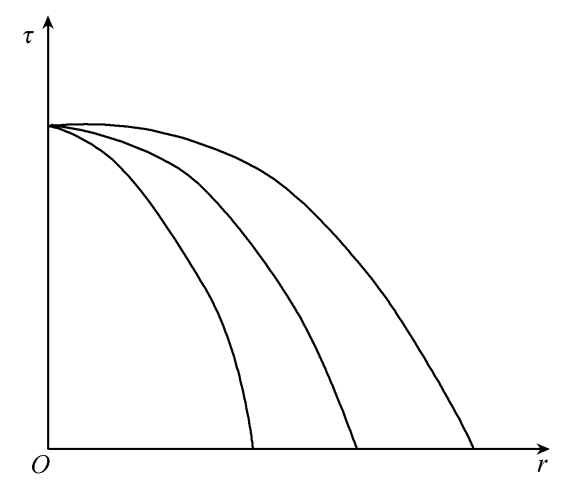

The online version of the original article can be found at http://dx.doi.org/10.1007/s11464006-0002-1 